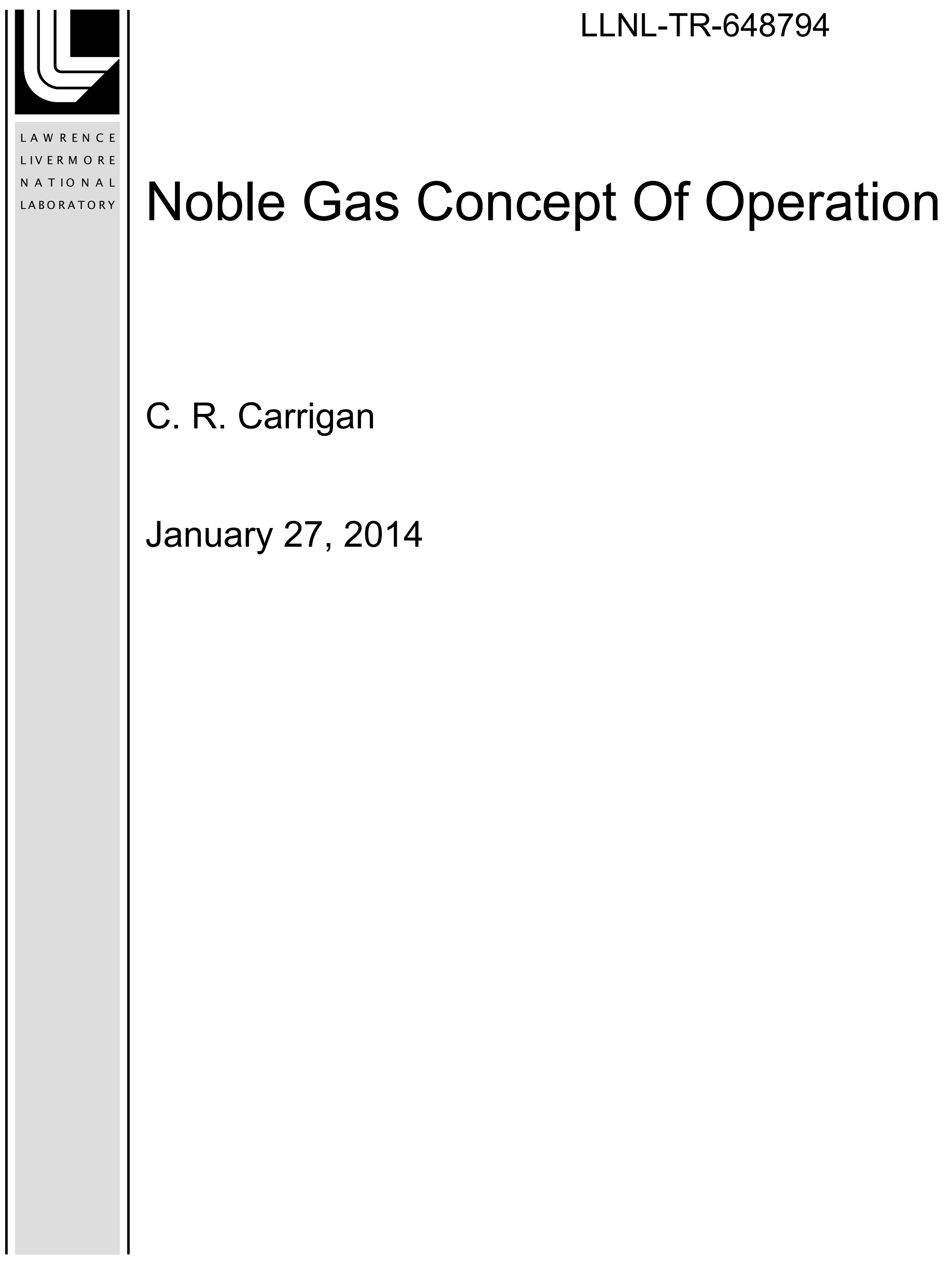


This document was prepared as an account of work sponsored by an agency of the United States government. Neither the United States government nor Lawrence Livermore National Security, LLC, nor any of their employees makes any warranty, expressed or implied, or assumes any legal liability or responsibility for the accuracy, completeness, or usefulness of any information, apparatus, product, or process disclosed, or represents that its use would not infringe privately owned rights. Reference herein to any specific commercial product, process, or service by trade name, trademark, manufacturer, or otherwise does not necessarily constitute or imply its endorsement, recommendation, or favoring by the United States government or Lawrence Livermore National Security, LLC. The views and opinions of authors expressed herein do not necessarily state or reflect those of the United States government or Lawrence Livermore National Security, LLC, and shall not be used for advertising or product endorsement purposes.

This work performed under the auspices of the U.S. Department of Energy by Lawrence Livermore National Laboratory under Contract DE-AC52-07NA27344. 


\title{
Noble Gas Concept Of Operation
}

\section{Detecting and Measuring Radioactive Noble Gases During an On-Site Inspection}

\author{
Charles R. Carrigan \\ $2 / 1 / 2014$
}

LLNL-TR-648794

This work was performed under the auspices of the U.S. Department of Energy by Lawrence Livermore National Laboratory under contract DE-AC52-07NA27344. Lawrence Livermore National Security, LLC

Disclaimer

The views expressed here do not necessarily reflect the views of the United States Government, the United States Department of Energy, or the Lawrence Livermore National Laboratory. 


\section{Contents}

Preface

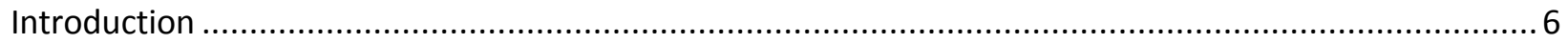

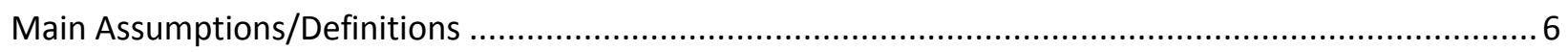

An underground nuclear explosion (UNE) is assumed to be the origin of noble gases of interest......... 6

Radioactive decay of noble gases of interest creates a "window" for detection by OSI operations....... 6

Atmospheric and subsurface background concentrations of noble gases of interest may exist potentially requiring differentiation from signatures associated with UNE detonation gases.

Subsurface noble gas sampling used for confirmation purposes and not search-area reduction unless

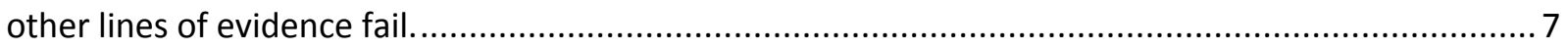

Noble gas air sampling is viable approach for search area (IA) reduction........................................ 7

Regions of interest (ROIs) are assumed to be potential UNE sites. ............................................... 7

Information input/output of noble gas CONOPS is fully integrated with other available lines of evidence (e.g., SAMS, visual observations, RN surveys, NG air survey, MSIR, CPT, etc.) ..................... 7

Information requested from the ISP on the location of all sources of radioactivity and recent NG

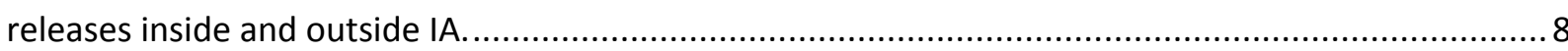

The noble gas CONOPS is intended to be applied within the context of the Team Functionality methodology developed by the CTBTO for carrying out an OSI. .................................................. 8

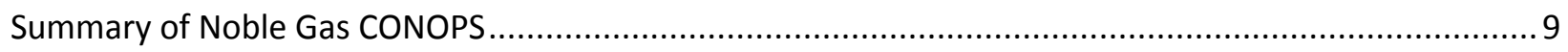

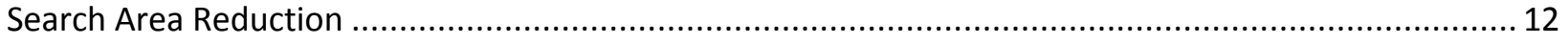

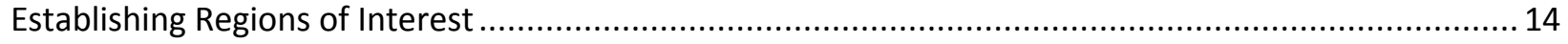

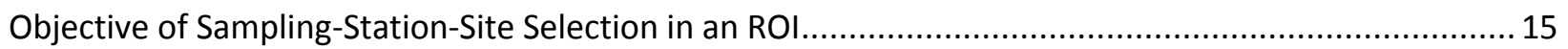

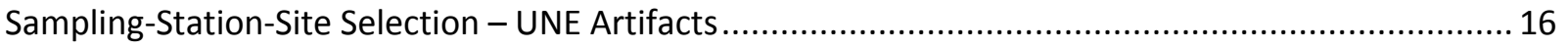

Sampling-Station-Site Selection - UNE Induced Pathways............................................................ 17

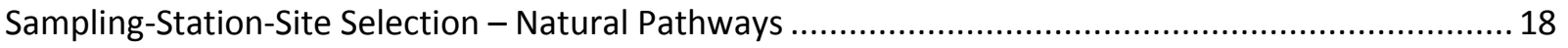

Sampling-Station-Site Selection - Featureless Site .................................................................. 19

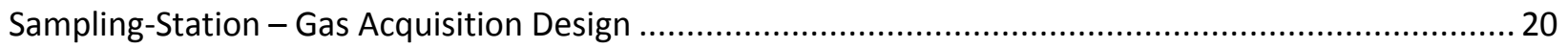

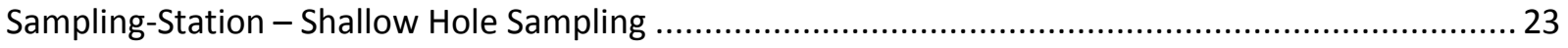

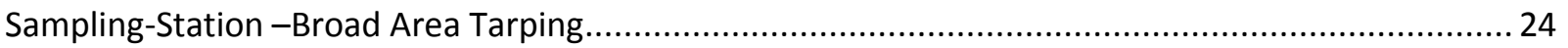

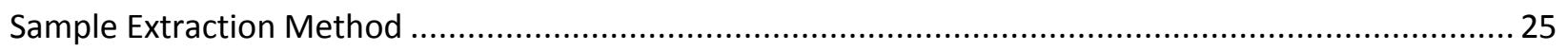

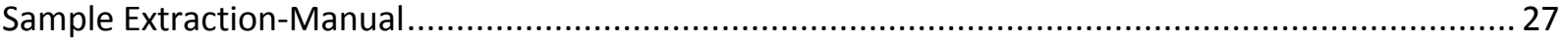

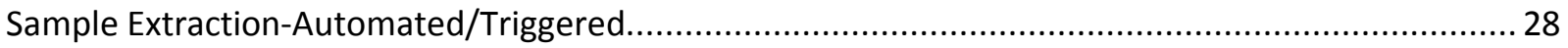

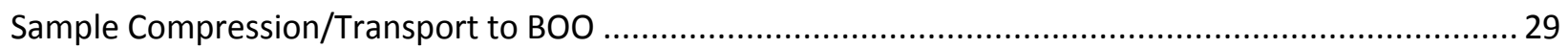




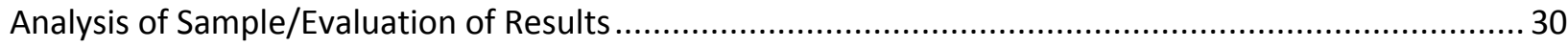

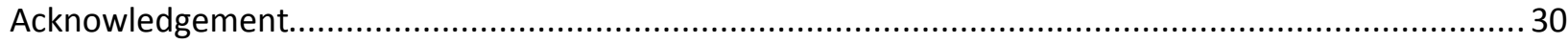

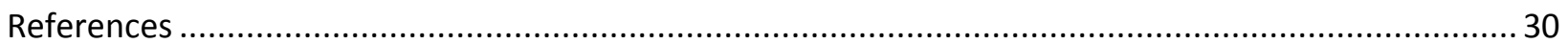




\section{Noble Gas Concept Of Operation}

\section{Preface}

The intent of this document is to provide the reader with an understanding of a general approach to performing the noble gas component of an On Site Inspection or OSI. The authors of this document recognize that owing to the wide range of scenarios that are possible for carrying out an underground nuclear explosion, the diverse sets of information that might be available to the inspection team initially and the potential range of political and physical constraints imposed during the inspection, a satisfactory prescriptive approach to carrying out the noble gas component of an OSI is unlikely. Rather, the authors intend only to aid the reader in understanding what a reasonable course of actions or responses may be as performed by an inspection team (IT) during a general OSI. If this document helps to inform the intuition of the reader about addressing the challenges resulting from the inevitable deviations from this general scenario, it will have achieved its intent. 


\section{Introduction}

We define a concept of operation (CONOPS) as a document describing the characteristics of a proposed system/methodology from the viewpoint of an individual who will use that system/methodology. A CONOPS may also be thought of as describing how a set of capabilities may be employed to achieve a desired objective or end state.

The primary objective of this CONOPS is the detection and measurement of radioactive noble gases during the course of an OSI released into the soil gas and/or atmospheric regimes for the purpose of determining the source of those gases [listed in Annex 1.4 of OSI Manual] that may be seeping, defusing, barometrically pumped or entrapped below the surface.

The reader is referred to the OSI Manual for additional context and to relevant standard operating procedures (SOPs) and work instructions (WINs) for additional details not provided here.

\section{Main Assumptions/Definitions}

\section{An underground nuclear explosion (UNE) is assumed to be the origin of noble} gases of interest.

Obviously, other possible sources of the noble gases of interest might exist (e.g., nuclear reactors, medical radionuclide production facilities, etc.), but UNEs are assumed to be the most likely origin of gases of interest. Thus it is assumed for this document that sites of interest are prospective underground explosion sites (e.g., Ref 1 and Ref 2, Fig. 1).

\section{Radioactive decay of noble gases of interest creates a "window" for detection by OSI operations.}

Half lives of radioactive noble gases and the fact that they may require some time to arrive at the surface following a UNE means that the possibility of detecting these gases exists within a temporal window of opportunity (e.g., Ref 2, Fig. 2). In some scenarios, seeps or venting of detonation gases may allow significant quantities to reach the surface and be released into the atmosphere immediately following a UNE. In other release scenarios, days to weeks may be required for gases to reach the surface at detectable levels (e.g., Ref 1).

\section{Atmospheric and subsurface background concentrations of noble gases of interest may exist potentially requiring differentiation from signatures associated with UNE detonation gases.}

A low-level, Argon-37 (Ar-37) natural background is produced in the shallow subsurface by interaction of cosmic ray neutrons with native calcium in the rock and soil (Ref. 3). Natural variations in the calcium content as well as in the hydrogeologic properties of the near-surface 
regime produce similar variations in the Ar-37 distribution with depth (Ref. 2, Fig. 11).

Atmospheric and soil-gas backgrounds of Xenon isotopes may occur owing to releases by nuclear reactors and medical isotope production facilities (Ref 2, Fig. 10). Fortunately, backgrounds of noble gases of interest tend to be low.

\section{Subsurface noble gas sampling used for confirmation purposes and not search-area reduction unless other lines of evidence fail.}

Subsurface gas sampling tends to be labor intensive and time consuming in regard to setting up subsurface sample stations, acquiring large volume $(2 \mathrm{cu} . \mathrm{m})$ samples, transporting them to the Base of Operations and finally performing analyses. However, for some OSI search-area reduction scenarios, subsurface gas sampling and analysis may be the only option for performing search-area reduction.

\section{Noble gas air sampling is viable approach for search area (IA) reduction.}

It is assumed that using portable noble gas atmospheric samplers may provide useful information for localizing or reducing the search area when used in conjunction with weather (e.g., wind direction) information. Any noble gas atmospheric signals will tend to have a timecritical nature. Therefore, fielding air samplers as early as possible during an OSI is highly recommended.

\section{Regions of interest (ROIs) are assumed to be potential UNE sites.}

In this CONOPS document, ROI is assumed to refer to a potential site where a UNE has occurred. Of course, other types of ROIs can be defined that possess OSI-relevant aspects requiring further investigation by the IT. Such ROIs would have to be eliminated from consideration during the search-area reduction process.

\section{Information input/output of noble gas CONOPS is fully integrated with other} available lines of evidence (e.g., SAMS, visual observations, RN surveys, NG air survey, MSIR, CPT, etc.)

It is assumed that relevant data from the different sub-teams and the Operational Support Center (OSC) is available to the RN sub-team, where SAMS refers to seismic aftershock monitoring, MSIR refers to multispectral infrared monitoring, and CPT refers to application of geophysical methods as continuation period technologies. This information is likely essential to localizing ROI in the first place and it is the fusion of all available information that will make for the most robust conclusions. 


\section{Information requested from the ISP on the location of all sources of radioactivity and recent NG releases inside and outside IA.}

This is an important point that should be part of most if not all scenarios. It is assumed here that any available noble gas release information (e.g., medical isotope production releases) will be requested from the inspected state party (ISP).

The noble gas CONOPS is intended to be applied within the context of the Team Functionality methodology developed by the CTBTO for carrying out an OSI.

No attempt is made in this document to define a decision tree that would be utilized to advance the search-area-reduction process or more generally to carry out an OSI. The overarching Team Functionality methodology already provides a logic framework including a decision tree for reducing the search area and for carrying out an OSI using information obtained during the course of activities carried out by the sub-teams. 


\section{Summary of Noble Gas CONOPS}

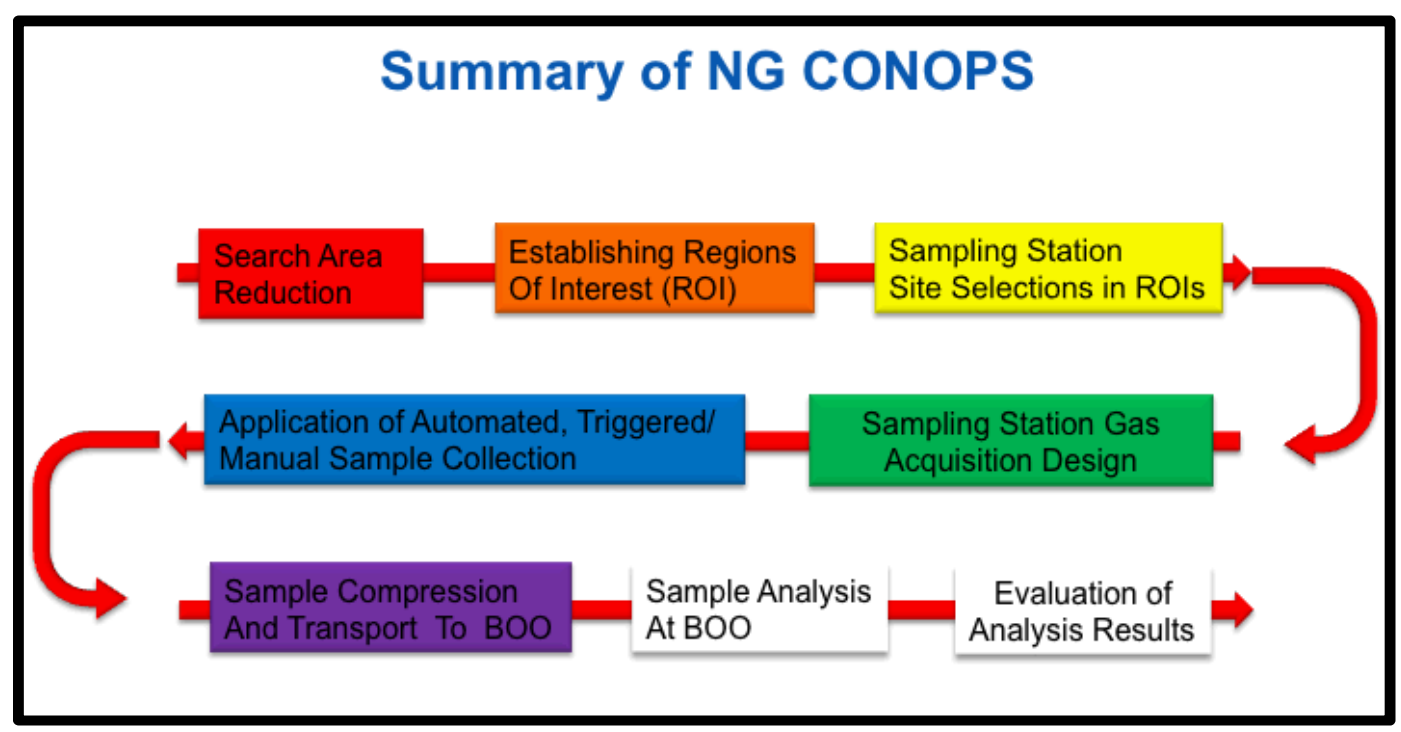

Figure 1. Diagram illustrating possible temporal sequence of the different elements of an OSI (i.e., Search Area Reduction, Establishing Regions of Interest, etc.) For some OSI scenarios, it may be reasonable to skip certain elements or groups of activities as determined by the Team Functionality methodology.

Given the assumption that a suspected UNE has occurred, Figure 1 illustrates a possible sequence of activities that could be pursued. Under the terms of the CTBT, a thousand-square$\mathrm{km}$ Inspection Area (IA) is initially allocated to be the search area. Input from inspector-led search area activities as well as pre-inspection information help guide the Search-Area Reduction process as identified in the first box (red) or element of Figure 1. Search-AreaReduction is a reduction in the area upon which search efforts are focused without identifying the most likely sites for a UNE. Under the Team Functionality methodology, it is possible to return to a low-likelihood area if new information increases its likelihood of containing a UNE site. For example, a populated locale exhibiting no signs of damage might reasonably be considered as a low-likelihood site for a recent UNE unless new information suggests otherwise.

Regions of interest or ROIs are zones within the remaining areas that are established as having the highest likelihood of being or containing a UNE site (Figure 1, orange box). ROIs are smaller, permitting more focusing of available inspection team resources. Multiple ROls may exist until information gathering and analysis is sufficient to eliminate all but the most likely ones.

Based on a variety of considerations including proximity to a suspected UNE site, inspectors will attempt to identify sites within an ROl offering the greatest potential to yield noble gases of interest sampled from the subsurface or from seeps into the above-ground environment (yellow box). Some understanding of gas transport from an underground detonation as well as 
the relationship between explosion-created pathways and natural ones (e.g., nearby faults) is helpful in selecting optimal locations for subsurface sampling in addition to criteria provided later on sampling site selection.

Once a sampling site is identified, the technique for acquiring the sample must be determined (Figure 1, green box). Features of the sampling site (e.g., rockiness, alluvium thickness, amount of moisture in the soil, etc.) tend to determine the method that is most appropriate for acquiring subsurface gases. Inserting sampling tubes into the subsurface or tarping the surface are two typical means of extracting gases from the subsurface. However, both subsurface sampling approaches are potentially subject to the possibility of contamination by atmospheric gases during large volume (e.g., cu. m) acquisitions. Obtaining above-ground atmospheric gas samples is one means of monitoring for the possibility of contamination (e.g., radioxenon isotopes) of soil gases by the atmosphere. Any atmospheric gas samples obtained to monitor for background contamination do not require analysis unless contemporaneously obtained subsurface gas samples indicate the presence of noble gases of interest.

Following identification of a sampling site, a decision is made regarding how the soil gas will be extracted (Figure 1, blue box). Either automated or manual sampling would be performed to extract a subsurface gas sample. Automated sampling has the advantage of not requiring an inspector to be present during sample acquisition which may occur owing to restrictions imposed by the Inspected State Party (ISP) or because of weather or environmental conditions are deemed inappropriate for health and safety best practices. Experience indicates that the best or optimal times for obtaining samples occurs during falling atmospheric pressure, which also corresponds to the onset of stormy, sometimes dangerous weather conditions. Automated sampling has the advantage of eliminating the need for human involvement in triggering the sampling process during the onset of a barometric low or some other sampling-activation indicator.

Currently, a gas sample (either subsurface or atmospheric) is extracted and stored temporarily in a low pressure tank or bladder until the sample can be transported to the Base of Operations (BOO) for analysis or storage. It has been found that compressing the sample into a SCUBA or compressed-air dive bottle significantly improves the transportability and storage of multicubic-meter samples (Figure 1, purple box).

After arrival at the BOO, analysis of the sample may occur or alternatively, the sample may be stored until needed. Enumeration of the steps required to analyze a sample at the BOO for noble gases of interest is beyond the scope of this document (Figure 1, white box). 
The evaluation of the sample results and determination of their significance is also beyond the scope of this document (final white box). Information from this CONOPS element becomes available to the Team Functionality methodology in directing the course of the OSI.

The list of activities as illustrated in Figure 1 is sequential and was constructed assuming what might be considered to be a typical OSI scenario. The activities listed in the boxes of Figure 1 assume a logical progression during the course of the scenario and are guided by the continuous acquisition and analysis of information relevant to the scenario. Obviously, deviations from the scenario and corresponding deviations from the list of activities are to be expected. As the noble gas concept of operation is intended for integration into the overarching Team Functionality methodology, deviations from the list of activities or their order of implementation will be controlled by that overarching methodology. 


\section{Search Area Reduction}

\section{$1000-S q-K m$ Search Area Reduction}

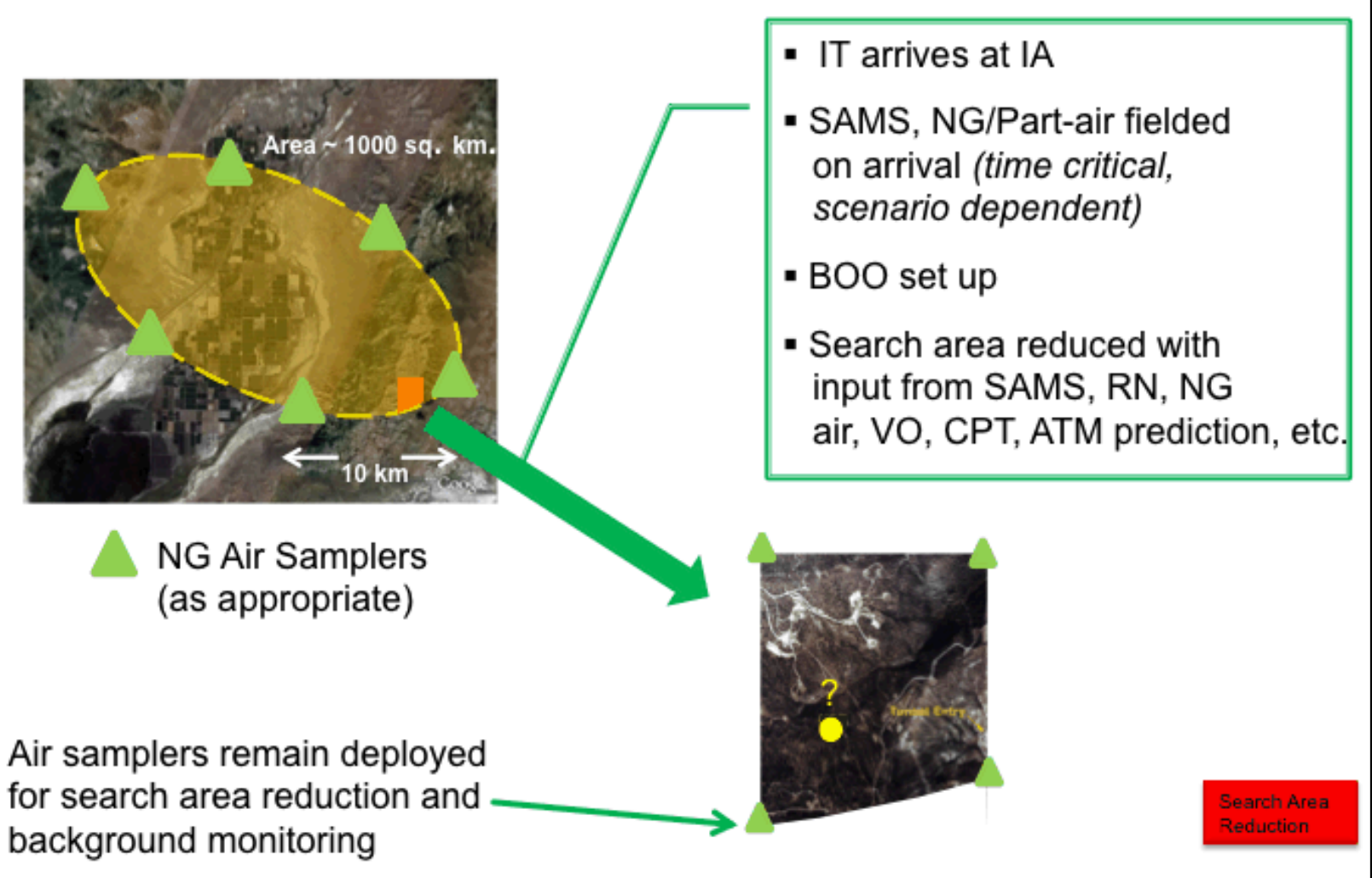

Figure 2. Example $1000 \mathrm{~km}^{2}$ search area reduction process.

On-site search area reduction activities may begin when the IT arrives at the initial 1000-sqkilometer IA. As illustrated in Figure 2, the initial search area is reduced in size with the aid of initial information and sub-team missions utilizing area-reduction methods such as SAMS, RN measurements (e.g., ground and allowed aerial surveys), Visual Observations (VO), Continuing Period Technologies (CPT) and predictive Atmospheric Transport Modeling (ATM) in conjunction with NG air sampling and particulate sampling.

Regarding noble gas air sampling during the search-area reduction process, immediate deployment of noble gas air samplers is carried out for the purpose of localizing sites of interest by back-projecting the atmospheric flow path of detected gases. Appropriate deployment of air sampling capabilities is subject to available wind-direction information, topographic considerations (e.g., distribution of hills/mountains, etc.), and any other information that may be relevant to the downstream placement of air samplers from the potential source of gas release. Because of the evanescent nature of UNE gas releases and the possibility that 
important search-area-reduction information will be lost, noble gas air samplers should typically be deployed as soon as possible after arrival in the IA to increase the possibility of detecting vented gases. The rapid deployment of noble gas air samplers following arrival in the IA is consistent with the rationale for the expedited deployment of the SAMS on arrival in the IA owing to the rapid fall off in time of post-detonation seismicity, or radiation signals in this case.

Another motivation for deploying noble gas air samplers is to continuously monitor the atmosphere for background levels of noble gases of interest. In particular, monitoring for detectable levels of noble gases of interest should typically be considered at the BOO where analyses are performed and also in the vicinity of subsurface soil gas sampling operations. Monitoring at the latter sites is recommended, given the potential for atmospheric gases to be captured during the soil-gas-sampling process. Generally, analysis for trace levels of noble gases in atmospheric samples may be deferred and archived until the analysis of soil gas samples produces a result requiring the evaluation of trace levels of those gases in the atmosphere to eliminate the possibility that the soil gas samples were contaminated by atmospheric infiltration of a site. Frequency and density of atmospheric monitoring in support of detecting air containing gases that might contaminate subsurface samples will necessarily include consideration of the availability of air sampling resources.

It will be seen that soil-gas sampling is a labor-intensive activity having a limited areal range of detection (i.e., typically $500 \mathrm{~m}$ depending on the underlying fracture network). Hence, other RN overflight and vehicle-based, VO and SAMS techniques are better suited for search-area reduction purposes while subsurface noble gas sampling is more applicable for the purpose of confirming the nature of the event. However, some scenarios may require the application of soil-gas sampling for search area reduction if other techniques fail.

Based on input from a variety of techniques employed by the sub-teams as well as other information utilized by the Team Functionality methodology, the 1000-sq-km search area is eventually reduced to a more tractable size from the perspective of noble gas subsurface sampling as illustrated in Figure 2. 


\section{Establishing Regions of Interest}

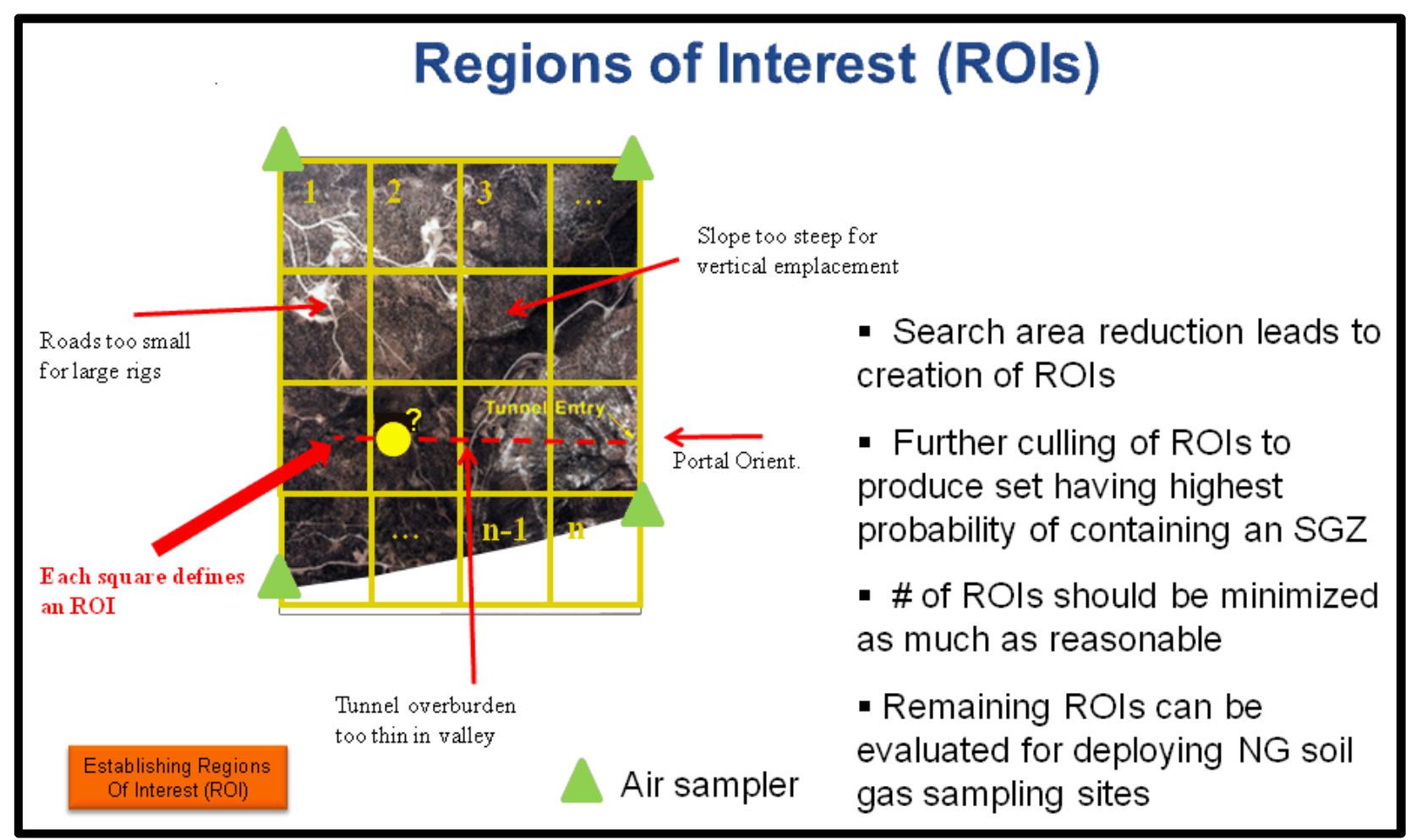

Figure 3. A reduced search area may consist of several smaller areas or just one as illustrated here. Within the area of this example are identified a number of ROIs corresponding to the boxes in the grid. In this particular example, it is assumed that an ROI contains a possible UNE site, but an ROI may also be thought to possess OSI-relevant aspects requiring further investigation by the IT.

The goal of this stage is to identify ROIs that might contain UNE sites. It is within these ROIs that noble gas subsurface sampling will be performed. Owing to the IT limitations of time, equipment and inspector availability, reducing the number of ROIs re quiring the application of subsurface noble gas techniques is a major objective of this stage of the noble gas operation. Note that air samplers may be in continuous use during this ROI reduction process as they may provide input to aid in eliminating some ROIs from further subsurface-sampling consideration. Also note that many other types of inputs may be required to both identify and eliminate ROls from immediate consideration. In the example of Figure 3., the orientation of a portal discovered in one of the ROIs along with the topography of others was used to eliminate some ROIs while raising interest levels in others. In this example, the process results a reduction from 16 to only several ROIs. The Team Functionality methodology controls the ROI establishment and reduction process with inputs from sub-team missions as well as other sources. 


\section{Objective of Sampling-Station-Site Selection in an ROI}

For subsurface noble gas sampling, the objective of the site selection activity is to use available surface (e.g., fractures, explosion or containment artifacts), near-surface observations (e.g., ground penetrating radar) and geologic considerations to specifically identify the UNE surfaceground-zero as well as nearby sites where subsurface pathways of UNE gas transport terminate at the surface. Ideally, some skill and knowledge relevant to performing the subsurface-gas sampling site selection process is required. In particular, understanding the potential relationship between explosion-produced pathways for gas transport and existing natural pathways is extremely helpful for identifying the most likely sites for the detection of UNEproduced noble gases.

In addition to understanding how the local geology and UNE containment practices may contribute to the existence of gas transport pathways, a similar understanding of how noble gas transport processes (i.e., barometric pumping, cavity pressurization and thermally driven convection) are responsible for noble gas detections obtained from subsurface gas samples is also highly desirable. Because such samples, which typically have large volumes ( $2 \mathrm{cu} \mathrm{m}$ ), tend to be collected very near the ground surface or even at the surface, familiarity with the mechanisms by which atmospheric dilution or contamination of a sample can occur (i.e., atmospheric gas infiltration into soil, leaks in sampling-hole sealant, barometrically driven soil gas memory effect, etc.) is of equal importance. The goal of this understanding is to select optimal sites for sampling that are also amenable to the application of techniques that minimize atmospheric dilution or contamination effects. For example, a fracture detected at the base of a soil layer may be a better candidate for producing a sample that is relatively undiluted by atmospheric infiltration than a readily visible surface fracture providing easy sample point installation and also an easy route for infiltration of the atmosphere to the sample point. 


\section{Sampling-Station Selection Criteria}

\section{UNE Testing/Containment Artifacts}

Possible sites containing UNE artifacts that may be directly linked to UNE detonation point:

- Emplacement casing

- Portal

- Wells/boreholes

- Cable bundles breaking surface Ventilation shafts Other suspect artifacts connected to subsurface

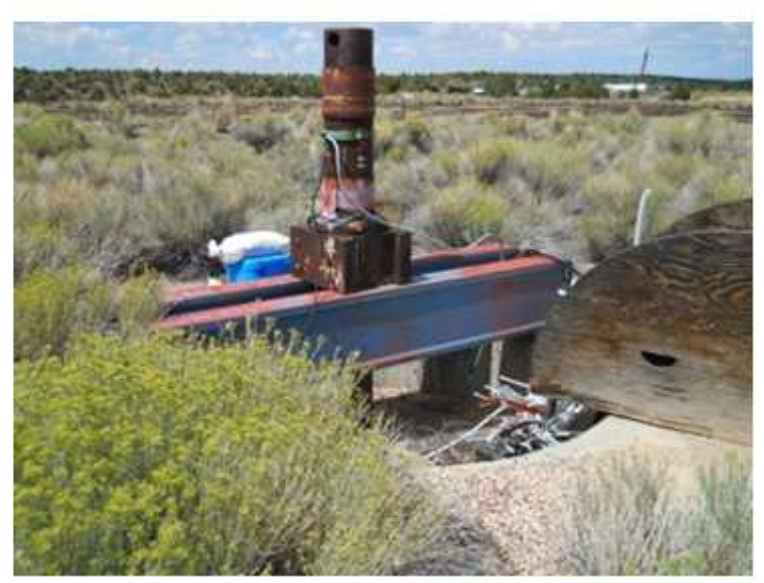

Sampling Station Site Selections in ROIs

Figure 4. Artifacts or objects associated with a potential UNE site that are connected to the subsurface regime are potentially excellent pathways for gases to reach the surface.

The Team Functionality methodology will be utilized with inputs from sub-team missions and other sources to progress toward identifying sites within an ROI that are promising for subsurface noble gas sampling. In the case of a UNE, which is assumed here, artifacts of the UNE may be discovered that not only represent evidence for the occurrence of a UNE but also function as pathways for gas transport to the surface. Such features as emplacement casing, portals of tunnels, boreholes and cables may be well connected to the subsurface and represent the greatest potential for producing at least forensic levels of noble gas signals. 


\section{Sampling-Station Selection Criteria}

\section{UNE-Induced Fractures \& Surface Changes}

\section{Possible explosion- produced changes observed at surface:}

- Cratering

- Radial fractures

- Circumferential fractures

- Linear fracture systems

- Down drops induced along local faults
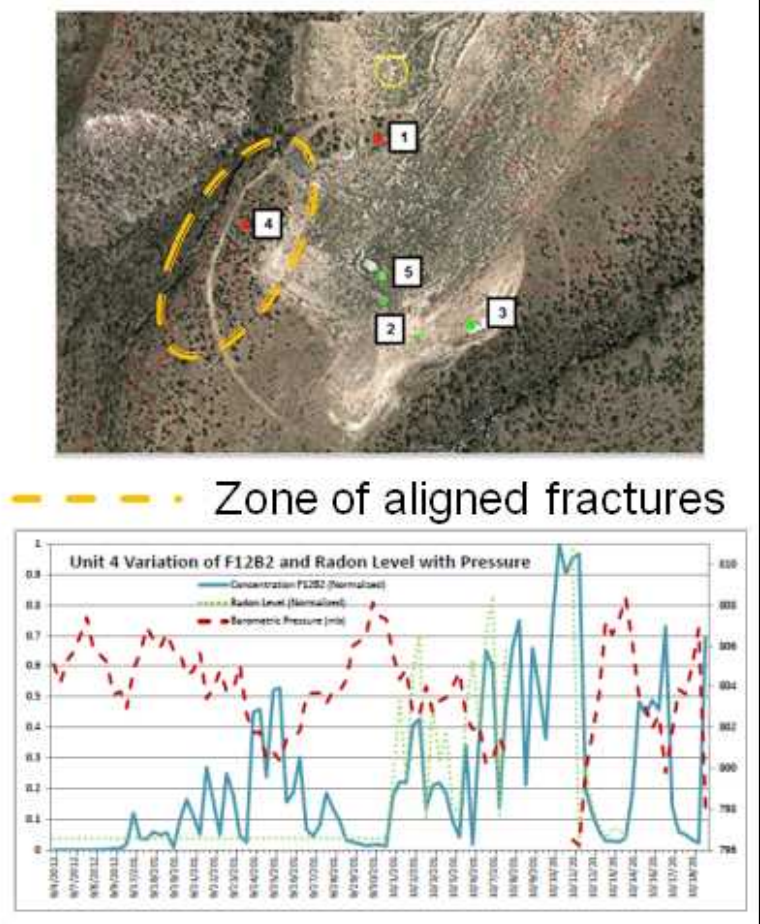

Figure 5. The UNE creates new pathways for subsurface gas transport. Cratering as a result of explosion-cavity collapse as well as fractures having different geometries are common features of a UNE although sufficiently deeply buried events may only exhibit very sub tle features or none at all. In the dashed ellipse of the overhead view of a UNE site, a gas-producing fracture resulted as a post-detonation feature. Note the strong anti-correlated behavior between the tracer concentration and the barometric pressure.

Besides manmade artifacts such as emplacement shafts, portals and cable bundles, UNE produced artifacts (e.g., fractures, reactivation of geologic faults) may produce excellent paths for gas transport to the surface. In rocky materials extensive subsurface fracturing tends to occur. Depending on the geology of the containment regime, fracturing may sometimes be enhanced. For example, the overhead view of the UNE site shown in Fig. 5 shows an ellipse surrounding a set of long, parallel fractures. The fractures occur near the edge of a terrace or down-drop that could not fully contain the outward motion of the underground detonation. As a result, underlying jointed columns of volcanic rock were slightly displaced horizontally creating this series of open parallel fractures that have produced strong gas signals during 
tracer experiments performed at the UNE site. The gas signals from the former detonation cavity/chimney system are shown in a plot that also includes barometric pressure fluctuations at the surface. Generally, a strong anti-correlation exists between the surface pressure fluctuations and the cavity gas signals measured at the surface.

\section{Sampling-Station-Site Selection - Natural Pathways}

\section{Sampling-Station Selection Criteria}

\section{Geologic/Natural Pathways}

Local geologic/natural pathways interconnected with potential explosion-induced fracture network:

- Natural fracturing at surface

- Nearby regional faults $(<500-$ $600 \mathrm{~m}$ from SGZ)

- Tree roots especially in thin alluvium over bedrock

Other features indicating subsurface fractures Sub-horizontal sedimentary layering with surface expression
Typical Emplacement Hole With Cooling Fractures
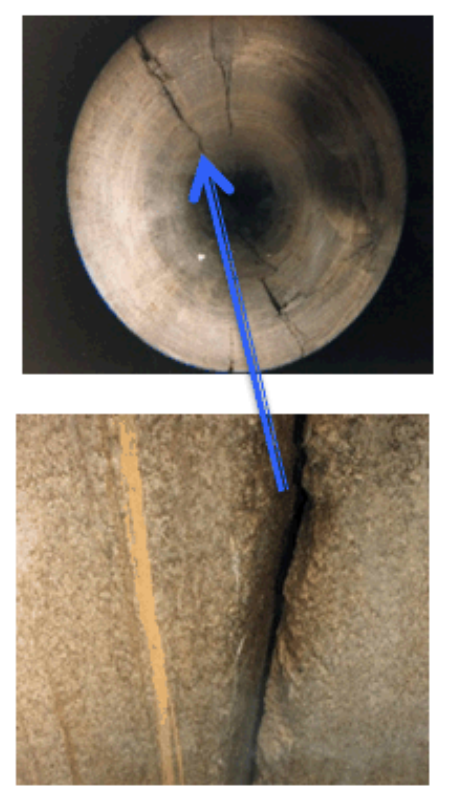

Sampling Station Site Selections in ROls

Figure 6. The Earth tends to be an imperfect container for UNEs as illustrated. Geologic pathways for gas flow occur in the form of cooling fractures in rock, local and tectonic faults and even sedimentary layering of sand or gravel. Such natural pathways form networks that may become interconnected with the explosion-produced fracture regime allowing transport of noble gases to the surface.

UNEs that are particularly deep for their level of yield may produce few fractures at the surface. However, the resulting explosively produced fracture systems may well interconnect with existing natural pathways allowing noble gases to be transported to the surface. Sampling along natural faults and fractures has produced detectable levels of gas transported from deep 
detonation points. Faults that may be reactivated locally by the explosion may form small down drops or offsets that represent ideal indicators of sites worth sampling using subsurface techniques.

\section{Sampling-Station-Site Selection - Featureless Site}

\section{Sampling-Station Selection Criteria}

\section{None of the Above!}

\section{When a suspected site is featureless on surface or does not fit any of the criteria 1-3:}

- Sample near/over suspected $S G Z$

- Other features indicating possible subsurface fractures

- Tree roots especially in thin alluvium over bedrock

Sampling Station Site Selections in ROIs

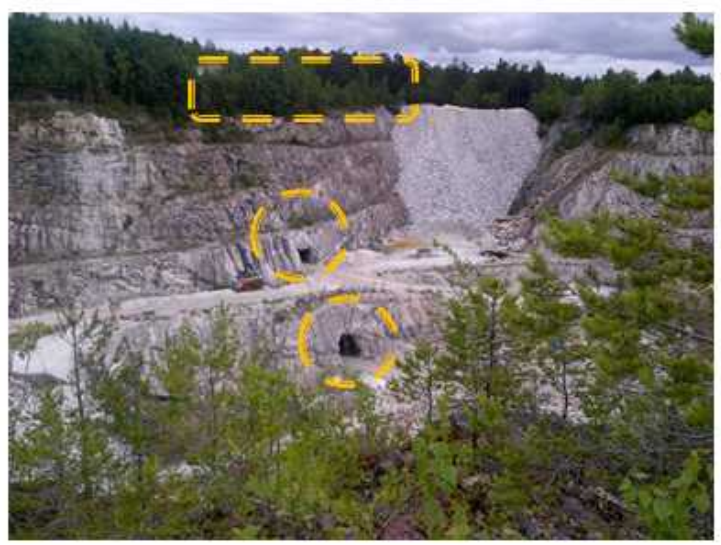

Possible sampling areas on surfaces above portals and tunnels. Treelines may indicate pathways where fractures are not apparent

Figure 7. Guidance in selecting sampling sites may be obtained from relational considerations (e.g., proximity to suspected surface ground zero as evidenced by portals or tunnels) and indirect indicators of pathways in shallow soil such as tree roots, especially if indicating damage from ground motion. In the case of evident portals and tunnels, gas sampling should also be performed if accessible.

The most difficult case for siting sampling stations occurs when the ground surface is essentially featureless except maybe for the presence of trees and other flora. In such cases, inspectors may only have the possibility of using the proximity to other more distant features (e.g., portals or tunnels below potential sampling areas as shown in Figure 7.) as indicators for establishing possible sampling stations. In some cases the distribution of flora (e.g., trees and bushes) could indicate the presence of a sharp subsurface division such as a fault that may serve as a pathway to the surface. Random sampling has been found to be far less effective for detecting signals than sampling associated with UNE artifacts or the geology of the site (e.g., faults) 


\section{Sampling-Station - Gas Acquisition Design}

Following sampling-site selection, a decision must be made concerning the type of subsurface sampling to be performed at each of the selected sites in a particular ROI. Two sampling designs have been previously employed in tracer experiments. They can produce comparable results and each has its advantages and disadvantages. The first method involves the insertion of a sample tube into the subsurface while the second method utilizes tarps or plastic sheeting placed on the surface over perforated or irrigation tubing through which gases reaching the surface are withdrawn. A major advantage of the sample tube approach is that samples are drawn directly from depth and the potential for atmospheric infiltration is reduced. The prevention of infiltration is particularly good when small, liter-sized samples are acquired but is less true with continuous sampling or when very large samples (e.g., $2 \mathrm{cu} \mathrm{m}$ ) are needed. To some degree, the atmospheric infiltration problem can be mitigated by the use of a tarp or plastic sheet laid on the surface and centered on the sampling tube that helps to minimize atmospheric gases from infiltrating through the soil near the sample tube to the gas-extraction sample point when large volumes are needed.

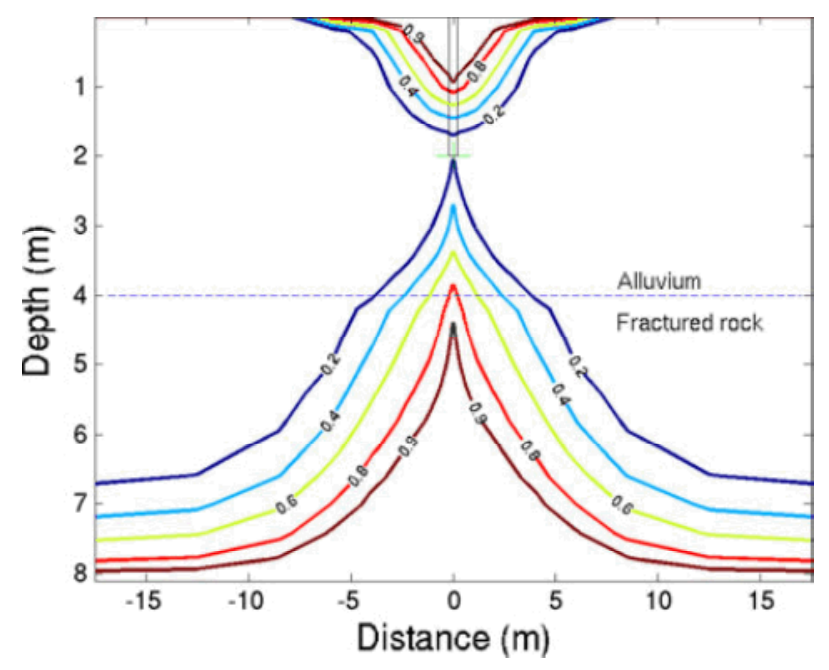

Figure 8a. Illustrates the effect of not using a ground sheet to prevent atmospheric infiltration to the sample extraction point at a depth of $2 \mathrm{~m}$. Not only is gas extracted from depth but there is also a large component of atmospheric gas captured as evidenced by the significant plume moving downward from the surface. (Ref. 2) 


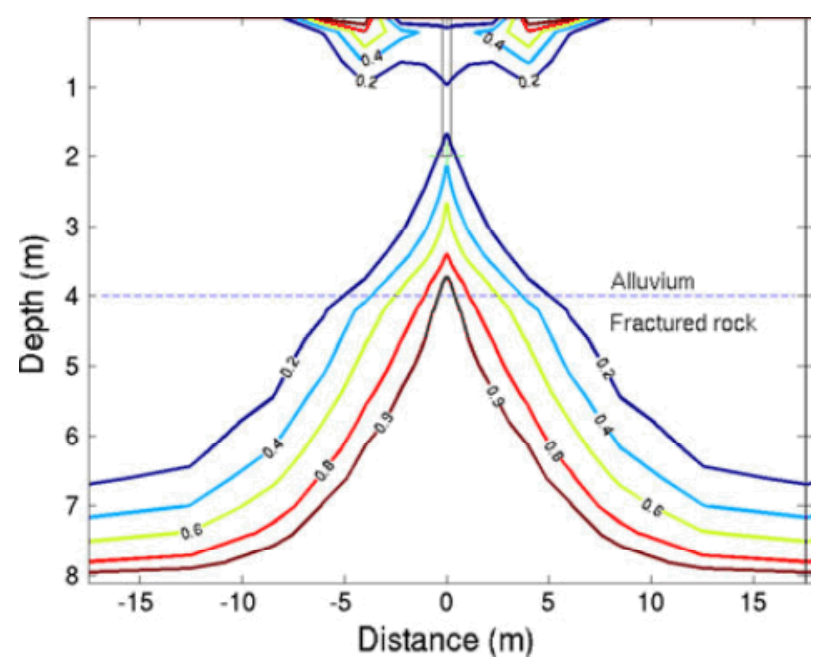

Figure 8b. Same gas extraction simulation but with a tarp or sheet laid around the sample tube. The infiltration plume is mitigated but still exists. Minimizing extracted volumes minimizes or eliminates the contributions of atmospheric infiltration. (Ref. 2)

Figures $8 \mathrm{a}$ and $\mathrm{b}$ show the effect of placing a tarp around the sample tube for an extraction point buried in alluvium at a depth of $2 \mathrm{~m}$. The $4 \mathrm{~m}$ alluvium overlies a zone of fracture rock. Extracting gas through the tube is seen to create plumes from below (i.e., the fractured rock with its potential noble gas signal) and from above (i.e., the atmosphere). The goal in sampling is to minimize or eliminate the plume from the atmosphere that can both dilute and contaminate the gases from the deep subsurface plume. Comparing the atmospheric plume in both Figure $8 a$ (no tarp) with that in Figure $8 b$ (tarp) shows that some mitigation of the atmospheric plume occurs but it is not eliminated for large volumes or continuous sampling. Additionally, the sealant (bentonite-water mixture) that is used around the sample tube after insertion in the augured hole potentially may leak allowing the direct flow of atmospheric gases to the subsurface extraction point. Fortunately, a means of continuously monitoring the sealing ability of the sampling hole exists using observations of the natural radon-gas background that will be considered later.

Finally, multi-point or distributed sampling of a feature (e.g., inserting multiple sample tubes spaced along a surface fracture) can reduce infiltration effects associated with large volume extractions by distributing the extracted volume among the sample points connected in common. The Smart Sampler allows up to 5 separate inputs, each having flow-rate control, for drawing a common sample simultaneously from multiple tubes. The multiple tube approach may also help to minimize any signal depletion effects that might result from excessive gas withdraw from a single sampling tube.

The second gas-acquisition method (tarp-and-tube) involves extraction of gases from beneath a tarp or plastic sheet placed on the surface over a feature or suspected feature of interest. 
Perforated tubing used in garden irrigation is looped beneath the tarp to extract gases coming to the surface. Sand or other permeable material is typically placed along the edges to hold the tarp down while allowing air to flow beneath it (Figure 9).

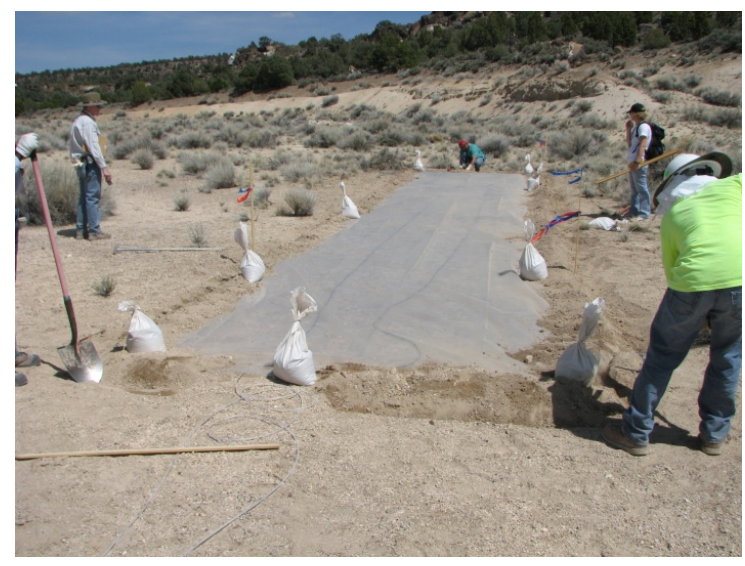

Figure 9. Tarp with underlying perforated tube. In this case dry soil is heaped up along the edges and rocks were placed in the center to minimize billowing during wind gusts. Tarp was emplaced over a suspected fracture system that was not directly observed. Its probable location was determined using post-detonation maps of the site and a GPS.

This sampling technique is of particular value when sampling an extended pathway regime or when some uncertainty exists concerning the location of a pathway (near-surface buried fractures). The technique also has value for continuous low-flow sampling of subsurface gases as signal depletion is not likely to be a problem as it potentially may be for the focused sampling associated with buried sample points.

A disadvantage of the tarp-and-tube sampling technique is the possibility of drawing atmosphere through the soil/sand piled at the edges of the tarp into the sampling zone beneath the tarp. This possibility must always be confronted during continuous sampling operations. However, sampling during a period of falling pressure may allow for samples to be acquired that have only a minimal atmospheric component. During falling atmospheric pressure gases are coming to the surface beneath the tarp. As the pressure continues to fall, gases will tend to build up beneath the tarp eventually flowing outward through the sand at the edges of the tarp. During periods when this occurs only gases from the subsurface are expected to be present beneath the tarp, providing an ideal regime for extracting soil gas samples. 


\section{Sampling-Station - Shallow Hole Sampling}

\section{Sampling-Station Gas Acquisition Design (1)}

\section{Shallow Hole Sampling Tubes}

\section{Sample points can be deployed at a feature of interest or site where desired and where direct push/augering is possible:}

- At least one sample point should be considered for a feature indicating a possible subsurface pathway

- Depth of sampling should ideally be greater than $2 \mathrm{~m}$ unless high-permeability layer or known fracture is shallower

- A plastic sheet should be used to minimize infiltration effects.

- Multiple sample points may be installed along long fractures/cracks with appropriate anti-infiltration sheeting

- Sampling tube installation is time and effort

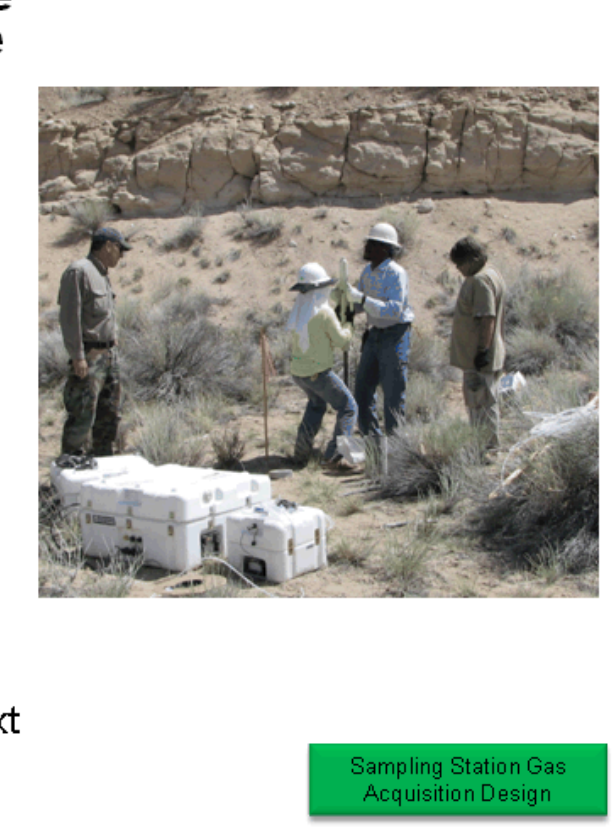
intensive which should be considered in context of resources when ranking best sites for subsurface sample-point deployment

Figure 10. Sampling tubes are most applicable when a feature of interest can be identified.

Sampling tubes are appropriate in situations where visible features can be identified such as cracks or depressions indicating cracks in the ground surface. The goal of sampling tube insertion is to emplace the sampling point as deeply as possible into the feature and usually more than $2 \mathrm{~m}$ unless a known high-permeability layer or fracture is shallower in which case the sample point should be emplaced in the vicinity of such subsurface features. Attempting to penetrate to depths greater than $2 \mathrm{~m}$ is necessary to minimize the contributions from the natural Ar-37 background which is anticipated to be at its maximum value at approximately this depth. To minimize the possibility of depleting a signal during a large volume sampling operation, it is advisable to insert multiple sampling tubes along a feature if possible. This procedure also increases the possibility of capturing a signal in the case where all sample points along a feature of interest are not equally productive. 


\section{Sampling-Station-Broad Area Tarping}

\section{Sampling-Station Gas Acquisition Design (2)}

\section{Broad-Area Tarping}

Plastic sheeting can be deployed at a feature of interest or site where desired if sample points are not deemed appropriate:

- Withdrawing soil gas from beneath a tarp tends to be unsuccessful for random tarp placements (e.g., unrelated to features)

- Water saturated soil is inappropriate for tarp sampling

- A minimum $3 \mathrm{~m} \times 3 \mathrm{~m}$ tarp/plastic sheet is suggested to minimize infiltration effects.

- After covering the multi-hole gas extraction tubing (irrigation) with sheeting, dry soil is heaped covering edges of plastic sheeting - Sand, rocks placed on tarp prevent billowing

- Tarping is also time and effort intensive which should be considered in context of resources when ranking best sites for

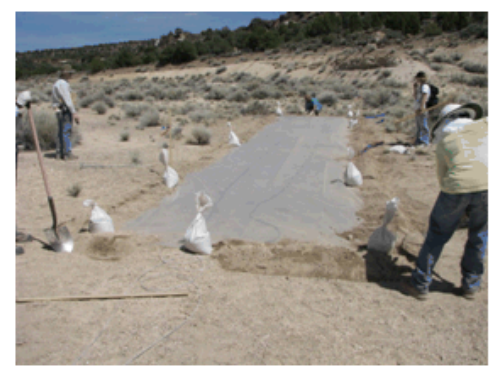
subsurface sample-point deployment

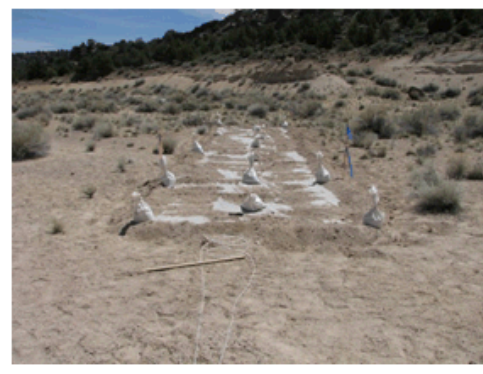

Sampling Station Gas Acquisition Design

Figure 11. Broad area tarping is appropriate when a tube cannot be easily inserted into a visible surface feature or there is some uncertainty about the location of a feature or pathway.

Broad area tarping has yielded good results when some evidence for a feature exists. Alternatively, random tarping can be attempted if an area is thought to contain potentially high-permeability pathways that are not visible at the surface and other possibilities for inserting sample points into or tarping over visible features are minimal. Soils with high levels of water saturation are generally poor hosts for extracting soil gases whether using sample tubes or tarps. The edges of tarps should not be buried in such soils as some communication between the area under the tarp and atmosphere is desirable. 


\section{Sample Extraction Method}

Sampling of subsurface gases tends to be performed at depths within a few meters of the ground surface or interface between the atmosphere and the subsurface. At such shallow sampling depths it is usually impossible to eliminate the effects of atmospheric pressure or barometric fluctuations on the sampling process. Additionally, it is known that a transport process known as barometric pumping is an important but slow mechanism (note: although fast compared to chemical diffusion) for moving gases from depth to the surface. Even if other transport mechanisms such as cavity pressurization temporarily dominate the transport of UNE cavity gases to the surface, barometric fluctuations are still observed to modulate the concentration levels of gases transported to the surface by an induced pressure gradient. Tracer-gas experiments generally show that a period of falling pressure is ideal for capturing gases of interest. When the barometric pressure is falling, the arrival at the surface of gases from depth is at a maximum and sampling that is timed to occur during a falling barometer has generally been found to produce the best results in terms of signal detection and the concentration of the gas of interest. Two general approaches to sampling exist and may be considered for extracting from sample sites identified in an ROI.

The first approach involves simple manual sampling using a gas pump and a collection bag. This is performed by inspectors possibly as part of a quick, early-stage survey of an ROI. In general, randomly timed grab samples have been found to produce results that are somewhat inferior to timing sample extractions by the onset of barometric lows, although very soon after a UNE, detectable signatures of interest may nevertheless be present at all times depending on the level of containment and persistence of subsurface transport mechanisms. However, consistent manual sampling during optimal periods may be difficult or impossible because the onset of a significant barometric low is often associated with a storm and it may not be possible for inspectors to be present in the IA during such times (Figure 12). Besides poor weather conditions, constraints placed by the ISP on entry into the IA or the general remoteness of ROIs in the IA may not permit inspectors to be present for sample acquisition during the best periods for sampling. 


\section{Sampling During Barometric Lows May Create Safety Concerns}
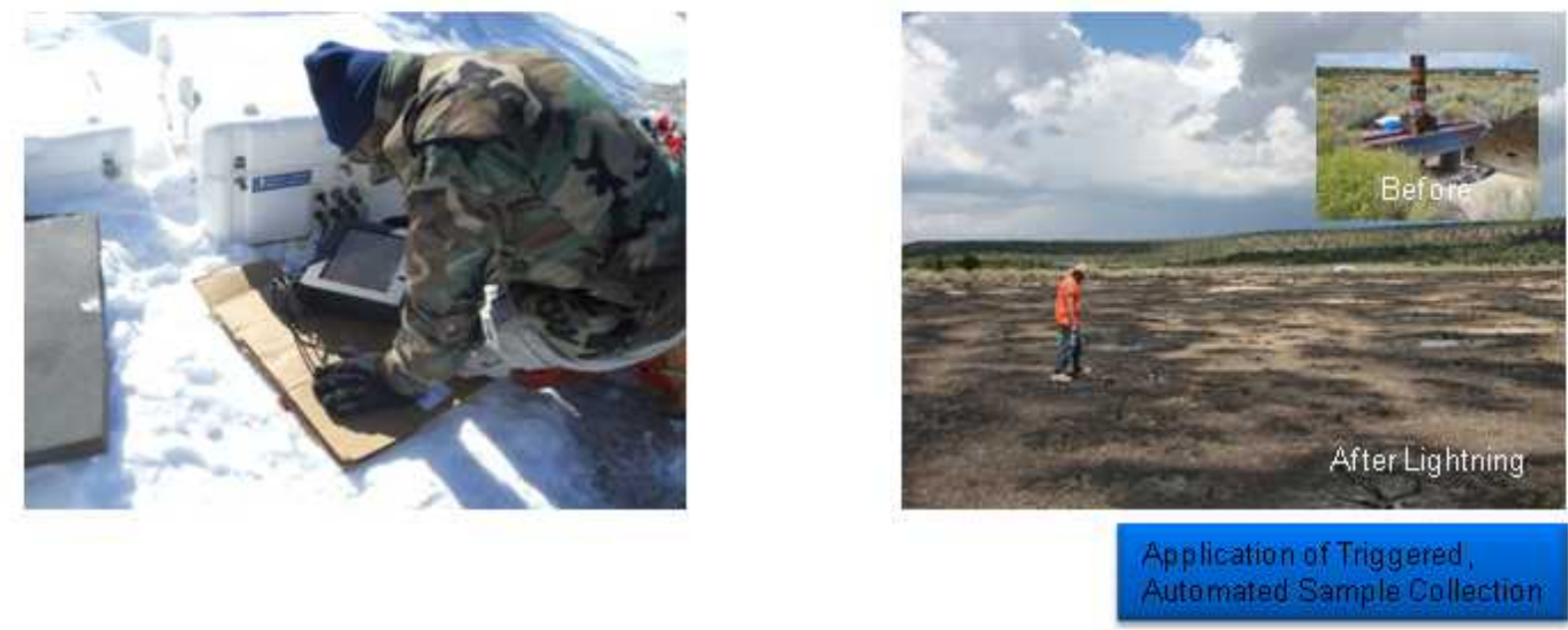

Figure 12. Barometric lows tend to be associated with the onset of storms. Automated sampling may be the only possibility for dealing with the requirement of sampling during poor weather.

To date, the best results for capturing signals have been achieved with an automated sampling approach that does not require human involvement. Several different automated sampling modes exist in the case of smart sampling systems. A continuous mode allows sampling all the time at some pre-set sampling or flow extraction rate. A barometric sampling mode can be selected that turns on the sampling system to a pre-set flow extraction rate only during a period of falling atmospheric pressure. Such periods are ideal for bringing gases from depth to the surface.

While sampling continuously may seem to have the advantage of capturing any gases of interest that might be present in the subsurface, gases of interest that have an evanescent nature will tend to be highly diluted by soil gases causing a reduction of any signature of interest potentially below meaningful levels. The barometric mode of sampling should produce a larger signal potentially resulting in detection of the gases at an earlier time during an OSI. Another advantage of sampling in the barometric mode is the minimization of atmospheric infiltration as a falling barometer produces outflow from the ground surface and not inflow.

A compromise mode that involves both continuous sampling and also takes advantage of the signature enhancement associated with the barometric mode is a barometrically-switched continuous mode of operation. In this mode, the sampling system switches between two 
sample storage bags or bladders depending on whether the barometer is falling or not. This mitigates concern that a critical signature might be missed during times when the sampling system may be idle, waiting to sample in the barometric mode. It also has the advantage of supplying a sample that is barometrically optimized. As with any continuous mode of sampling a significant disadvantage is the potential for high levels of atmospheric infiltration causing dilution and in some cases contamination of the sample. Another disadvantage is the production of more samples than can be readily analyzed at the BOO. However, implementation of a triage approach to selecting samples for analysis may reduce the impact of having more samples than can readily be analyzed. Other modes of automated sampling also have been proposed (e.g., background radon triggered) but these are still considered in the experimental stages of development and are not considered further here.

\section{Sample Extraction-Manual}

\section{Manual Sample Acquisition}

\section{Manual sampling is appropriate for capturing gases from subsurface anytime a grab sample is desired for site/signature evaluation:}

- Atmospheric or subsurface gas grab samples may be obtained

- Adjustable extraction rate up to 20 liters/min to match extraction requirements in field

- Volume totalizer to determine total sample size acquired
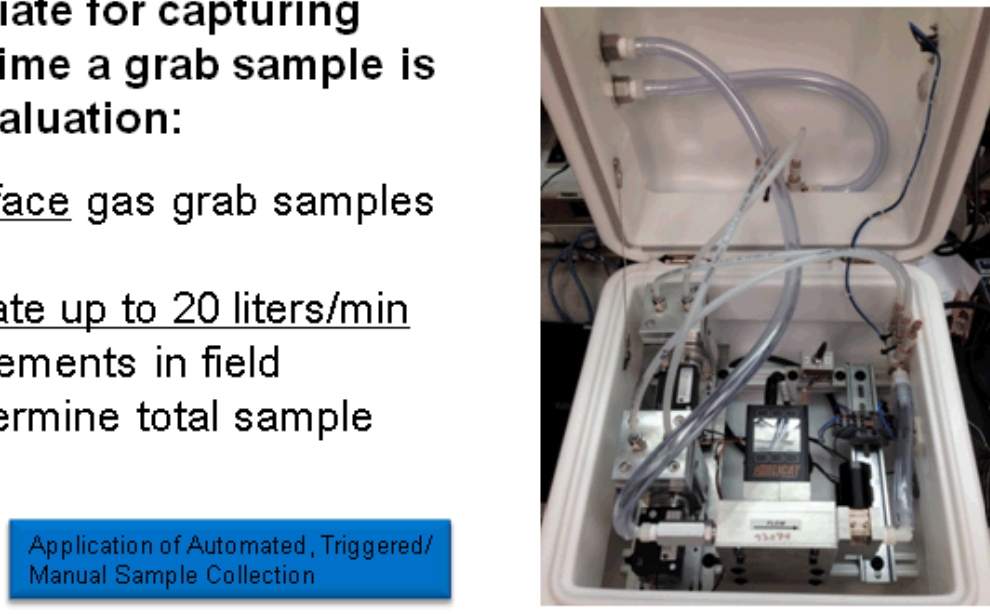

Figure 13. Manual sampler is highly portable being readily transported by one inspector. At least one $12-\mathrm{V}$ battery is required to power this system.

When inspectors can be present to sample within the IA during optimal times for sampling or simply when a grab sample is desired, a manual sampler as illustrated in Figure 13 should meet most field requirements. The basic unit can extract gases and determine the extraction rate as well as the total volume extracted. However, the unit has no capability to monitor the state of the sampling station for documenting the quality of a particular subsurface gas extraction. 


\title{
Automated Sample Acquisition: Triggering
}

\author{
Triggered sampling is appropriate for \\ capturing gases from subsurface using either \\ sample tubes or tarps:
}

- Barometric triggering used to sample during falling barometer to obtain maximized NG signal

- Continuous sampling/Barometric switching samples continuously switching between storage bladders as barometer rises and falls

- Radon monitoring is experimental but may have application for triggering and monitoring for leaks

- Pressure-drop measurements allow monitoring for changes in extraction process indicating potential quality issues

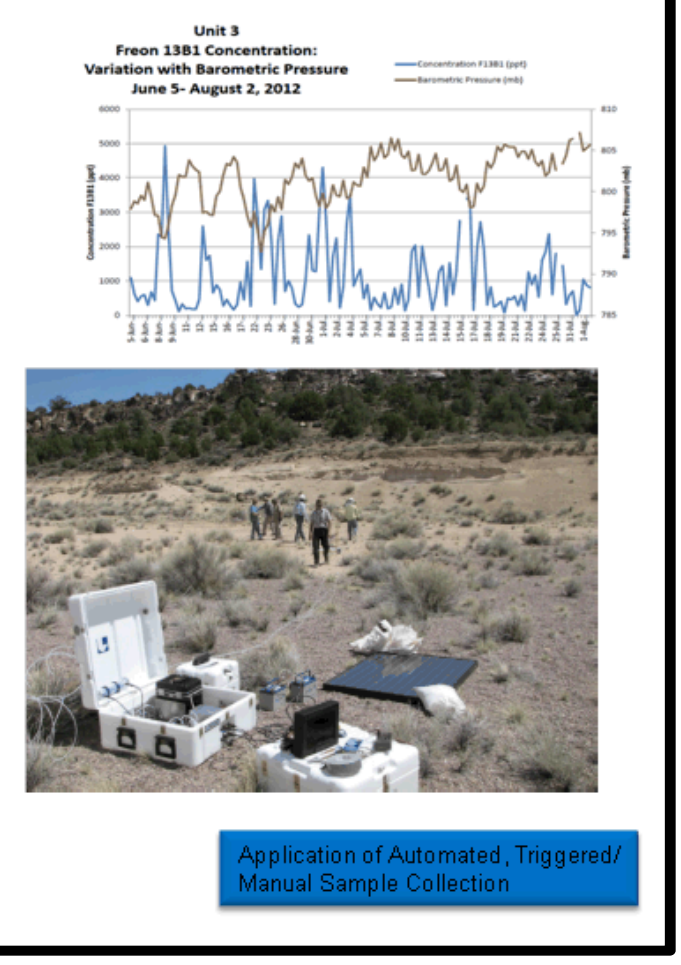

Figure 14. The automated smart sampling system shown is capable of operating in a number of extraction modes as indicated. In addition it allows some level of diagnostic information to be recorded during the sampling process which may be useful for evaluating the quality of a sample, for example, whether or not atmospheric leaks were significant.

The automated smart sampling system that is currently in use is a suit-case-sized unit that is still portable and can be carried over uneven ground easily by two people (Figure 14.). The unit has five rather than the manual sampler's one sampling input readily allowing distributed subsurface sampling to be performed. Each of the five input sampling lines can be separately set for flow rate and individually monitored for flow rate, total volume extracted per line and flow pressure. In addition, the radon level of the total stream can be monitored with time. Both the radon levels and pressure measurements can be used to infer the state of health of the sampling site during the sampling process. For example, a rapid decline in radon levels may indicate the existence of a leak or a sudden increase in atmospheric infiltration during the sampling process. If a pressure sensor also shows a sudden decrease in line pressure required for a given flow rate, this supports the interpretation of a leak in one of the sampling lines or in the sealing of a sample point itself. Because the pressure is monitored in each of the smart sampler's five lines, it is then possible to identify the line or sample point in which the leak may be occurring. The smart sampler periodically records its location using an internal GPS system 
and also monitors for physical shocks as well as attempts to open it - two features pertinent to maintaining its physical security.

The smart sampler is intended to be straightforward to program from a touch-screen monitor. Connecting the touch screen externally allows programming or resetting of sampling parameters to be performed without opening the sampler unit, which is desirable to avoid during extreme weather. Besides the gas samples taken, sampler and sampling station data can be downloaded externally to a laptop or to a memory stick.

While the smart sampler can also be used to obtain grab samples, it was designed to function unattended at a site of interest during the course of a sampling campaign. It can be powered by car batteries, a small portable generator or by solar panels.

\section{Sample Compression/Transport to B00}

\section{Gas Compression And Transport}

\section{After smart sampler collects gas sample in a low pressure bladder/bag, gas is compressed into SCUBA bottles that are then transported to $\mathrm{BOO}$ :}

- Typical volumes compressed are

$2 \mathrm{cu} \mathrm{m}$. at $1 \mathrm{~atm}$ pressure

- Compression times are about 20-30 minutes

- Compressed gas at -3000 psi

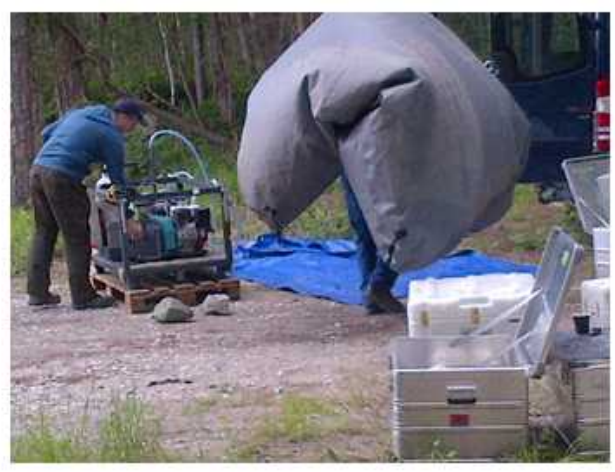

Low pressure gas in bag prepared for compression into SCUBA bottles

Figure 15. Compression of the extracted gas from the atmospheric pressure bladder or accumulator into SCUBA bottles is the final step prior to transport to the BOO.

The samples are stored in low-pressure bladders or inflatable cells while being acquired. Currently, gas in the bladders is compressed into high-pressure SCUBA bottles using a gasolinepowered diving compressor (Figure 15). Using an appropriate chain-of-custody methodology, the samples in the bottles can be properly associated with information relevant to the sample such as the log created by a smart sampler during the course of acquiring a gas sample. The samples may then be transported to the BOO for triage, analysis or archiving. 


\section{Analysis of Sample/Evaluation of Results}

Detailed discussion of these elements of the Noble Gas Concept of Operation is beyond the scope of this document. However, the resulting output will be utilized by the Team Functionality methodology to determine future mission needs in conjunction with input from other OSI subteams. One important role of both the sampling, analysis and evaluation elements of the CONOPS will be to determine characteristic background values of the gases of interest in order to determine the significance of the levels that are measured as inherent geo-hydrologic variability will likely affect background levels significantly even in the same ROI.

\section{Acknowledgement}

The author wishes to thank Steve Kreek (LLNL), Nathan Wimer (LLNL), Jerry Sweeney (LLNL) and Laura Marsh (OSD/DoD) for their helpful reviews of this work. Production of this document was made possible by the DoE NNSA Office of Nuclear Verification (NA-243). Many ideas presented here are based on the results of experiments supported through National Security Technologies, LLC by the DoE NNSA Office of Proliferation Detection (NA- 221).

\section{References}

1. CARRIGAN, C.R., HEINLE, R.A., HUDSON, G.B., NITAO, J.J., ZUCCA, J.J.: Trace gas emissions on geological faults as indicators of underground nuclear testing, Nature, 382(6591), 528-531, 1996.

2. CARRIGAN, C.R. and SUN, Y.: Detection of noble gas radionuclides from an underground nuclear explosion during a CTBT on-site inspection, Pure Appl. Geophys., 2012, doi: 10.1007/s00024-012-0563-8

3. RIEDMANN, R., and PURTSCHERT, R.: Natural ${ }^{37}$ Ar concentrations in soil air: Implications for monitoring underground nuclear explosions, Environ. Sci. Technol., 45(20), 8656-8664, 2011.

4. SUN, Y. and CARRIGAN, C.R: Modeling noble gas transport and detection for the Comprehensive Nuclear -Test-Ban Treaty, Pure Appl. Geophys., 2012. doi: 10.1007/s00024-0120514-4 